\title{
Screening for the genes involved in bombykol biosynthesis: identification and functional characterization of Bombyx mori acyl carrier protein
}

\section{Atsushi Ohnishi *, Misato Kaji, Kana Hashimoto and Shogo Matsumoto*}

Molecular Entomology Laboratory, RIKEN Advanced Science Institute, Wako, Japan

\section{Edited by:}

Joe Hull, USDA Agricultural Research

Service, USA

Reviewed by:

Honoo Satake, Suntory Institute for Bioorganic Research, Japan

Sheng Li, Chinese Academy of Sciences, China

Shunsuke Moriyama, Kitasato

University, Japan

*Correspondence:

Atsushi Ohnishi and Shogo

Matsumoto, Molecular Entomology Laboratory, RIKEN Advanced Science Institute, Hirosawa 2-1, Wako,

Saitama 351-0198, Japan.

e-mail: aohnishi@riken.jp;

smatsu@riken.jp

\begin{abstract}
Species-specific sex pheromones released by female moths to attract conspecific male moths are synthesized de novo in the pheromone gland (PG) via fatty acid synthesis (FAS). Biosynthesis of moth sex pheromones is usually regulated by a neurohormone termed pheromone biosynthesis activating neuropeptide (PBAN), a 33-aa peptide that originates in the subesophageal ganglion. In the silkmoth, Bombyx mori, cytoplasmic lipid droplets (LDs), which store the sex pheromone (bombykol) precursor fatty acid, accumulate in PG cells prior to eclosion. PBAN activation of the PBAN receptor stimulates lipolysis of the stored LD triacylglycerols (TAGs) resulting in release of the bombykol precursor for final modification. While we have previously characterized a number of molecules involved in bombykol biosynthesis, little is known about the mechanisms of PBAN signaling that regulate the TAG lipolysis in PG cells. In the current study, we sought to further identify genes involved in bombykol biosynthesis as well as PBAN signaling, by using a subset of 312 expressed-sequence tag (EST) clones that are in either our B. mori PG CDNA library or the public $B$. mori EST databases, SilkBase and CYBERGATE, and which are preferentially expressed in the PG. Using RT-PCR expression analysis and an RNAi screening approach, we have identified another eight EST clones involved in bombykol biosynthesis. Furthermore, we have determined the functional role of a clone designated BmACP that encodes B. mori acyl carrier protein (ACP). Our results indicate that BmACP plays an essential role in the biosynthesis of the bombykol precursor fatty acid via the canonical FAS pathway during pheromonogenesis.
\end{abstract}

Keywords: bombykol, Bombyx mori, lipid droplet, triacylglycerol, acyl carrier protein

\section{INTRODUCTION}

In most moth species, sex pheromone biosynthesis is regulated by the pheromone biosynthesis activating neuropeptide (PBAN), a neurohormone consisting of 33 amino acid peptide that originates in the subesophageal ganglion and that is characterized by the core C-terminal FSPRLamide sequence. Initially purified and sequenced from the corn earworm, Helicoverpa zea (Raina et al., 1989) and the silkmoth, B. mori (Kitamura et al., 1989), PBAN has since been identified in numerous other lepidopteran species (Rafaeli and Jurenka, 2003). In most species including B. mori, PBAN is released into the hemolymph during a species-specific period after adult emergence and acts on the pheromone gland (PG) to trigger the production and release of species-specific sex pheromones (Rafaeli, 2002, 2009). The PG is a functionally differentiated organ in close proximity to the terminal abdominal tip which originates in the intersegmental membrane between the eighth and ninth abdominal segments (Percy and Weatherston, 1974; Fónagy et al., 2000; Matsumoto et al., 2007). In the silkmoth, Bombyx mori, the sex pheromone, bombykol ( $E$, $Z$-10, 12-hexadecadien-1-ol), is synthesized de novo within PG cells from acetyl-CoA via conventional long chain fatty acid synthesis (FAS; Bjostad et al., 1987; Jurenka, 2003). The straight chain fatty acyl intermediate, palmitate, is converted stepwise to bombykol by the actions of a bifunctional Z11-10/12 fatty acyl desaturase, Bmpgdesat1, and a PG-specific fatty acyl reductase, pgFAR (Moto et al., 2003, 2004; Ohnishi et al., 2006). On the day before adult emergence, $B$. mori PG cells rapidly accumulate numerous lipid droplets (LDs) within the cytoplasm (Fónagy et al., 2001). These LDs play an essential role in bombykol biosynthesis by acting as a reservoir for the de novo synthesized bombykol precursor, 10,12-hexadecadienoate, which is deposited in the LDs in the form of triacylglycerols (TAGs) with the precursor predominantly sequestered at the $s n-1$ and $s n-3$ positions of the glycerides (Matsumoto et al., 2002). After adult emergence, the stored fatty acid is cleaved and converted to bombykol in response to PBAN stimulation (Matsumoto et al., 2007, 2010).

To identify functional proteins involved in bombykol biosynthesis, we generated a PG expressed-sequence tag (EST) database by constructing a normalized PG cDNA library prepared from newly emerged female $B$. mori moths of the inbred p50 strain (Yoshiga et al., 2000). We next screened in silico for PG-specific genes, using our own database as well as the public B. mori EST databases (SilkBase and CYBERGATE). Of the approximate 11,000 independent EST clones, we identified a number of clones that are 
predominantly or specifically expressed in the B. mori PG. Apart from this type of approach, we also established a method of RNAimediated in vivo gene silencing in B. mori (Ohnishi et al., 2006). Using this methodological tool, we have characterized a number of genes that are specifically or predominantly expressed in the $B$. mori PG during pheromonogenesis. These genes, which are up-regulated on the day prior to adult emergence (i.e., $p g F A R$, Bmpgdesat1, pgACBP, mgACBP, PBANR, BmFATP, and BmLsd1), play critical roles in bombykol production triggered by the external PBAN signal (Matsumoto et al., 2001; Moto et al., 2003, 2004; Hull et al., 2004; Ohnishi et al., 2006, 2009, 2011). In the current study, we describe the further identification of eight EST clones involved in bombykol biosynthesis using similar methodologies. Among these clones, we have also determined the functional role of a clone (NRPG0659) that encodes the B. mori acyl carrier protein (BmACP) critical for bombykol biosynthesis.

\section{MATERIALS AND METHODS INSECTS}

Larvae of the inbred p50 strain of $B$. mori, originally provided by T. Shimada of the University of Tokyo, were reared on an artificial diet and maintained under a 16L:8D photoperiod at $25^{\circ} \mathrm{C}$ (Fónagy et al., 1992). Pupal age was determined based on morphological characteristics as described (Matsumoto et al., 2002).

\section{RAPID AMPLIFYING cDNA ENDS (RACE)}

Messenger RNA was isolated from about $100 \mu \mathrm{g}$ of PG total RNA by using a Micro-Fast Track kit (Invitrogen). RACE was performed using a GeneRacer kit (Invitrogen) according to the manufacturer's instructions. Computer-assisted sequence analyses were performed using GENETYX-MAC Ver.15.0.5 (Software Development Co., Tokyo, Japan).

\section{RT-PCR ANALYSIS}

Pheromone glands and other tissues were dissected into insect Ringer's solution [ $35 \mathrm{mM} \mathrm{NaCl}, 36 \mathrm{mM} \mathrm{KCl}, 12 \mathrm{mM} \mathrm{CaCl}_{2}, 16 \mathrm{mM}$ $\mathrm{MgCl}_{2}, 274 \mathrm{mM}$ glucose, and $5 \mathrm{mM}$ Tris ( $\mathrm{pH} 7.5$ )] and mechanically trimmed as described (Ozawa and Matsumoto, 1996). Total RNA was isolated from the trimmed PGs by the method of Chomczynski and Sacchi (1987). First-strand cDNA synthesis was performed with $500 \mathrm{ng}$ total RNA using an RNA PCR kit (Takara Bio, Otsu, Japan) according to the manufacturer's instructions. Fragments of BmACP and Actin were amplified using a specific oligonucleotide primer set designed from the published sequence [for BmACP, NRPG0659-F (sense primer): 5' GCACAATTAAAGCCGCTGTC-3' ${ }^{\prime}$ and NRPG0659-R (antisense primer): $5^{\prime}$-ATGTCTTTTGGTCTGACCAG-3' ; for Actin, Actin-F (sense primer): $5^{\prime}$-AGATGACCCAGATCATGTTTG- $3^{\prime}$ and Actin$\mathrm{R}$ (antisense primer): 5'-GAGATCCACATCTGCTGGAAG-3'). PCR was performed using thermacycler conditions consisting of 30 cycles at $94^{\circ} \mathrm{C}$ for $30 \mathrm{~s}, 55^{\circ} \mathrm{C}$ for $30 \mathrm{~s}$, and $72^{\circ} \mathrm{C}$ for $60 \mathrm{~s}$. PCR products $(5 \mu \mathrm{L})$ were electrophoresed on a $1.5 \%$ agarose gel in Tris-acetate-EDTA buffer and stained with ethidium bromide.

SYNTHESIS AND INJECTION OF DOUBLE-STRANDED RNA (dsRNA)

Templates for the synthesis of dsRNAs corresponding to $B m A C P$ were prepared using gene specific primers containing
T7 polymerase sites [NRPG0659-T7-F (sense primer): $5^{\prime}-\underline{\mathrm{C}}$ CGGATCCTAATACGACTCACTATAGGGCGGCACAATTAA-3', NRPG0659-T7-R (antisense primer): 5' -CCGGATCCTAATACGA CTCACTATAGGGCGCCAATACATTGC-3'; nucleotide sequences corresponding to the T7 promoter region are underlined]. PCR was performed using thermacycler conditions consisting of six cycles at $94^{\circ} \mathrm{C}$ for $30 \mathrm{~s}, 56.5^{\circ} \mathrm{C}$ for $30 \mathrm{~s}, 68^{\circ} \mathrm{C}$ for $90 \mathrm{~s}$ followed by 30 cycles at $94^{\circ} \mathrm{C}$ for $30 \mathrm{~s}, 66^{\circ} \mathrm{C}$ for $30 \mathrm{~s}, 68^{\circ} \mathrm{C}$ for $90 \mathrm{~s}$ using KODPlus - (Toyobo, Osaka, Japan) with the resulting products purified (Wizard SV Gel and PCR Clean-Up kit, Promega) and used as templates to generate dsRNAs using the AmpliScribe ${ }^{\mathrm{TM}}$ T7 High Yield Transcription kit (Epicentre Technologies, Madison, WI, USA) according to the manufacturer's instructions. After synthesis, the dsRNAs were diluted with diethyl pyrocarbonate (DEPC) - treated $\mathrm{H}_{2} \mathrm{O}$, the RNA concentrations measured $\left(\mathrm{Abs}_{260}\right)$, and the products analyzed by gel electrophoresis to confirm annealing. Samples were diluted to the desired concentration (final volume $2 \mu \mathrm{L}$ ) and injected into 1-day-old pupae (i.e., pupae 1 day removed from the larval-pupal molt) using a $10 \mu \mathrm{L}$ microsyringe (Hamilton, USA) as described (Ohnishi et al., 2006; Hull et al., 2009, 2010). Control pupae were injected with $2 \mu \mathrm{L}$ DEPC-treated $\mathrm{H}_{2} \mathrm{O}$ alone. After injection, pupae were maintained under normal conditions until adult emergence.

\section{IN VIVO BOMBYKOL ANALYSIS}

Adult females were decapitated within $3 \mathrm{~h}$ of emergence and maintained at $25^{\circ} \mathrm{C}$ for $24 \mathrm{~h}$. They were then injected with either $2 \mu \mathrm{L}$ $(2.5 \mathrm{pmol} / \mu \mathrm{L})$ B. mori PBAN in PBS or PBS alone. Abdominal tips were dissected 90 min after injection and bombykol production was measured by HPLC as described (Matsumoto et al., 1990) using a Senshu-Pak $\mathrm{NO}_{2}$ column [Senshu Scientific Co., Tokyo, Japan; $4.6 \mathrm{~mm}$ inner diameter $\times 150 \mathrm{~mm}$, pore size, $100 \AA$ )].

\section{MICROSCOPIC EXAMINATION OF CYTOPLASMIC LDs}

PGs were dissected and mechanically trimmed from normal, decapitated, and RNAi-treated females. The excised glands were fixed with a $4 \%$ formalin/PBS solution and stained with Nile Red (a fluorescent probe for intracellular neutral lipids; Molecular Probes, Eugene, OR, USA) as described (Fónagy et al., 2001). Fluorescence microscopy was performed with an OLYMPUS BX-60 system equipped with a PM-30 exposure unit and a BH20-RFLT3 light source (400× magnification). Nile Red imaging was done with a $330-385 \mathrm{~nm}$ band pass excitation filter, a $400 \mathrm{~nm}$ dichroic mirror, and a $420 \mathrm{~nm}$ long pass barrier filter (Olympus cube WU). Images were processed and merged using Photoshop CS (Adobe Systems).

\section{ANALYSIS OF LD TRIACYLGLYCEROLS}

Lipid droplets TAGs were analyzed as described previously (Matsumoto et al., 2002). Five trimmed PGs were prepared from the desired stages of female moths and dipped in $100 \mu \mathrm{L}$ acetone for $10 \mathrm{~min}$ at room temperature. The dried acetone extracts were dissolved in $n$-hexane and loaded on either a Senshu-Pak PEGASIL-Silica 120-5 column (Senshu Scientific Co.; $4.6 \mathrm{~mm}$ inner diameter $\times 250 \mathrm{~mm}$, pore size, $120 \AA$ ) equilibrated with $n$-hexane/acetic acid (99/1) or a SSC-C 22 docosil column (Senshu Scientific Co.; $4.6 \mathrm{~mm}$ inner diameter $\times 250 \mathrm{~mm}$, pore size, 
$120 \AA)$ equilibrated with acetonitrile/ethanol (6/4). Because various TAG components comprise the cytoplasmic LDs, the entire TAG fraction was separated and detected using an evaporative light scattering detector (ELSD; SEDEX model 75, Sedere, France). Fatty acyl groups in the TAGs were identified by fast atom bombardment mass spectrometry (FAB-MS) and tandem MS (MS/MS) analyses using a JMS HX/HX-110A tandem mass spectrometer (JEOL, Tokyo, Japan) as described (Matsumoto et al., 2002).

\section{RESULTS \\ EXPRESSION ANALYSIS OF B. MORI EST CLONES USING RT-PCR}

To identify proteins functionally involved in bombykol biosynthesis, we selected $88 \mathrm{PG}$-specific genes in silico from approximately 11,000 independent clones in our PG EST database and/or the public B. mori EST databases. To examine the in vivo expression patterns of the 88 gene transcripts, we performed RTPCR analysis using various tissues including PG, which were prepared from B. mori females (p50 strain) before and after eclosion. The results indicated that 26 clones were specifically expressed in the PG and/or up-regulated the day prior to eclosion
(Table 1), suggesting potential functional roles for these genes in pheromonogenesis.

\section{RNAi SCREENING FOR GENES INVOLVED IN B. MORI PHEROMONOGENESIS}

We previously established a method of RNAi-mediated in vivo gene silencing in B. mori (Ohnishi et al., 2006). To confirm the in vivo function of the 26 genes described above, we performed dsRNA-mediated RNAi knockdown of these target genes and examined their inhibitory effect on bombykol production. Compared to control pupae injected with DEPC-treated $\mathrm{H}_{2} \mathrm{O}$, 1-dayold p50 female pupa injected with $10 \mu \mathrm{g}$ dsRNA corresponding to the respective genes resulted in a significant reduction in bombykol production for 8 of the 26 genes (NRPG0074, NRPG0604, NRPG0659, NRPG0660, NRPG0873, NRPG0891, NRPG1860, and NRPG1885; Figure 1). Because no decrease in bombykol production was observed in control pupae injected with dsRNAs for unrelated proteins including enhanced green fluorescent protein (EGFP), these findings suggest that disruption of bombykol production results from specific knockdown of the target gene sequences (Figure 1). These eight EST clones include two clones

Table 1 | Expressed-sequence tag clones identified in the Bombyx mori pheromone gland.

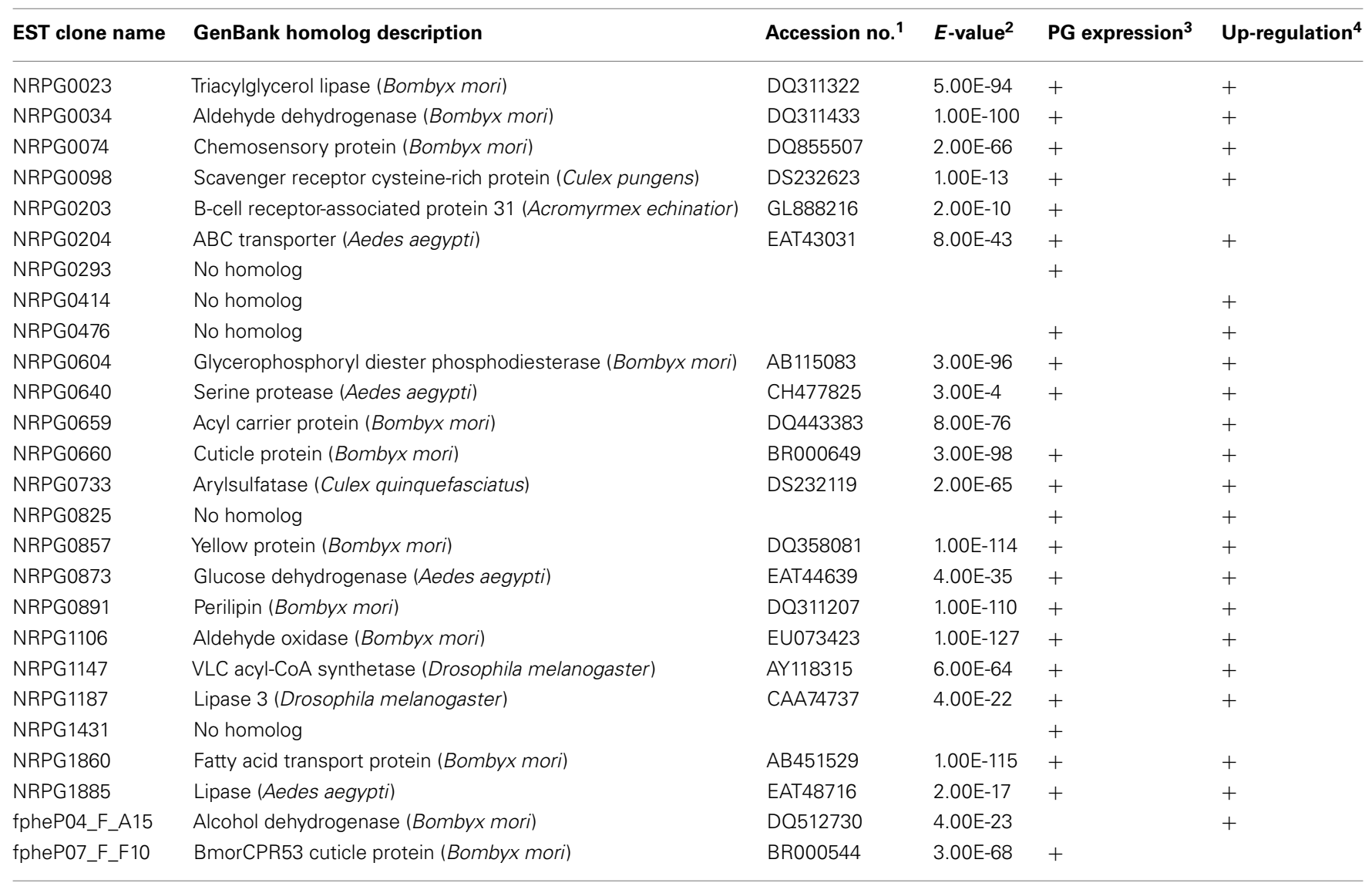

${ }^{1}$ Accession number of the GenBank homolog.

${ }^{2} E$-value for the comparison of the EST sequence information and the GenBank homolog (BLASTX).

${ }^{3}+$ Indicates a gene that is predominantly expressed in the $P G$.

${ }^{4}+$ Indicates a gene that is up-regulated from the day prior to eclosion in the $P G$ 


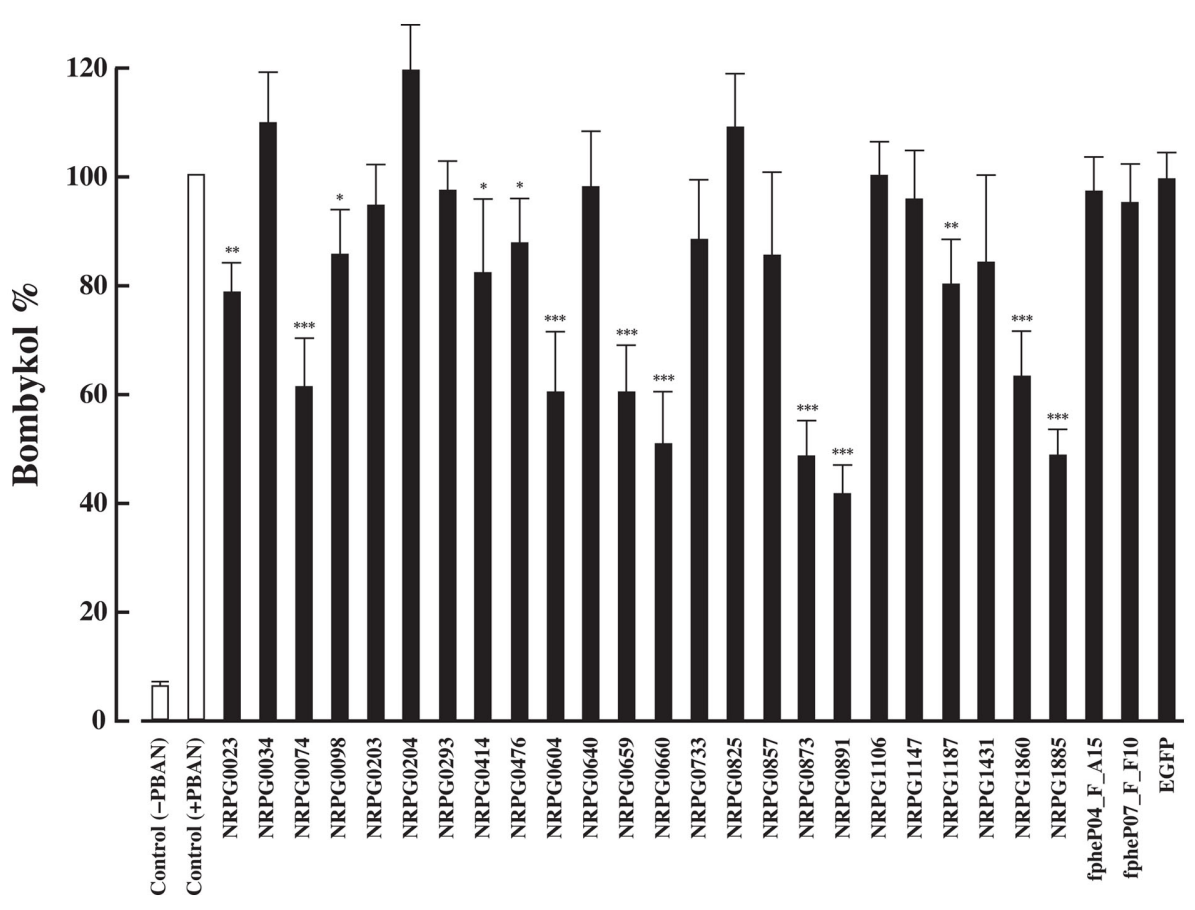

FIGURE 1 | Effect of RNAi treatment on in vivo bombykol production. One-day-old pupae were injected with $10 \mu \mathrm{g}$ dsRNA corresponding to the target genes or EGFP. Results are expressed relative to the mean value for bombykol levels in non-RNAi-treated females following injection with $5 \mathrm{pmol}$ PBAN ( ${ }^{*} p<0.05 ;{ }^{* *} p<0.01 ;{ }^{* *} p<0.001$ ). Bars represent mean values \pm SD from independent experimental animals $(n=>15)$.

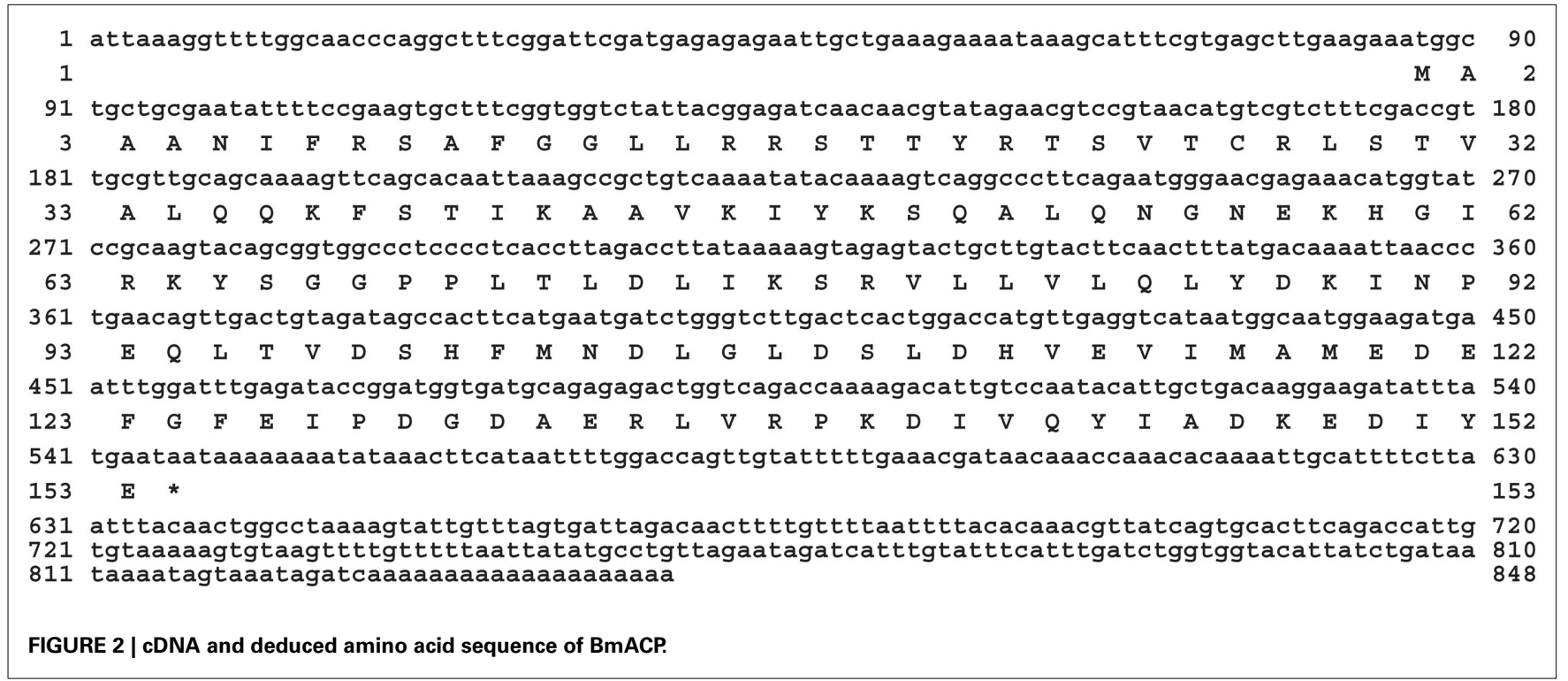

that encode BmLsd1 and BmFATP (NRPG0891 and NRPG1860), which we have recently characterized functionally associated with LD dynamics during B. mori pheromonogenesis (Ohnishi et al., 2009, 2011). From this group, five of the genes (NRPG0604, NRPG0659, NRPG0891, NRPG1860, and NRPG1885) are predicted to encode proteins involved in lipid metabolism (Table 1), which is consistent with a role in bombykol biosynthesis. The roles of the other three genes, which encode a chemosensory protein
(NRPG0074), a cuticle protein (NRPG0660), and a glucose dehydrogenase (NRPG0873), in bombykol biosynthesis have yet to be identified.

\section{CHARACTERIZATION OF NRPG0659}

Our RNAi-mediated gene silencing screen suggested the involvement of NRPG0659 in bombykol production (Figure 1). NRPG0659 is predicted to encode an acyl carrier protein (Table 1), 


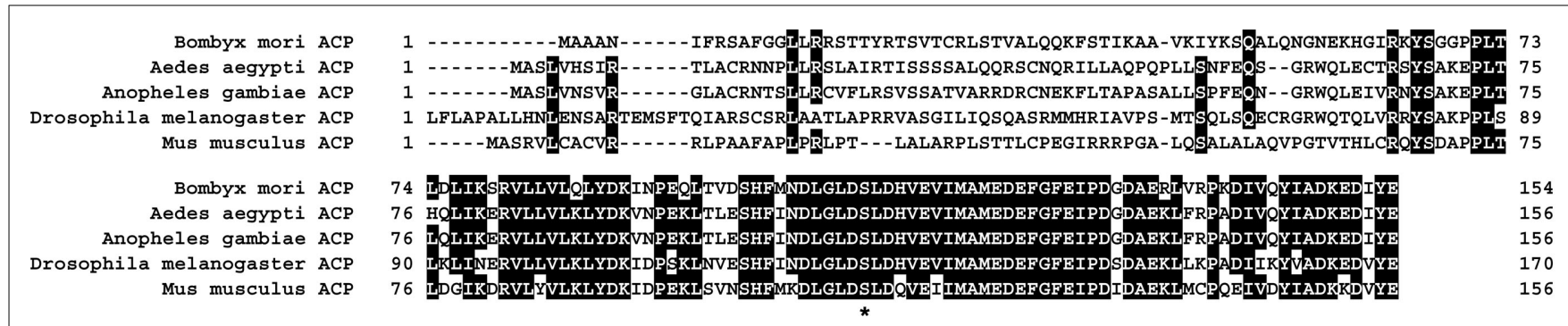

FIGURE 3 | Multiple sequence alignment of BmACP and related proteins. The predicted protein sequence of BmACP is compared with those of the Aedes aegypti, Anopheles gambiae, Drosophila melanogaster, and Mus musculus proteins (GenBank accession numbers: DQ443383, EAT36207,
EAA07190, ADB57059, AAH92379). Numbering of each sequence begins at the predicted initiator methionine. Amino acid residues identical to the $B$. mori sequence are highlighted in black. The asterisk $(*)$ indicates the prosthetic group attachment site (Ser). which is known to be a universal and highly conserved carrier of acyl intermediates during FAS (Byers and Gong, 2007). Consequently, we sought to characterize the functional role of the BmACP expressed in the PG. Structural analysis using both $3^{\prime}$ and $5^{\prime}$-RACE confirmed that BmACP is comprised of a 848nt ORF encoding a 153 -aa protein flanked by a 85 -nt $5^{\prime}$-UTR and a 304-nt $3^{\prime}$-UTR (Figure 2). Comparison of the BmACP sequence with sequences from other organisms revealed high similarities with the Aedes aegypti ACP, Anopheles gambiae ACP, Drosophila melanogaster ACP, and Mus musculus ACP (59, 59, 54, and 54\% similarity, respectively; Figure 3). These ACPs including BmACP are characterized by a highly conserved Cterminal domain sequence that contains a conserved serine residue necessary for interacting with acyl proteins (Figure 3; Byers and Gong, 2007). While RT-PCR analysis revealed that BmACP is ubiquitously expressed in adult tissues (Figure 4A), it undergoes up-regulation 1 day prior to adult emergence in the $\mathrm{PG}$ (Figure 4B). This up-regulation profile is reminiscent of PG proteins crucial to bombykol biosynthesis (Matsumoto et al., 2001; Moto et al., 2003, 2004; Hull et al., 2004; Ohnishi et al., 2006, 2009, 2011).

\section{IN VIVO EFFECT OF RNAi-MEDIATED BmACP KNOCKDOWN ON BOMBYKOL PRODUCTION}

To confirm the in vivo function of BmACP, we returned to dsRNAmediated RNAi knockdown and examined the effect on transcript levels. When we injected $10 \mu \mathrm{g} B m A C P$ dsRNA into newly emerged adult p50 females, $B m A C P$ mRNA levels 2 days after injection in the $\mathrm{PG}$ were selectively reduced compared to those in other tissues as well as control females injected with DEPC-treated $\mathrm{H}_{2} \mathrm{O}$ alone (Figure 5A). Although the reason for the PG-selective knockdown of BmACP is presently unknown, a similar PG-selective decrease in transcript levels was observed following knockdown of BmFATP and BmLsd1 (Ohnishi et al., 2009, 2011). Because BmACP dsRNA injections had no effect on pupal development or adult emergence, we next injected varying concentrations $(1,5$, and $10 \mu \mathrm{g})$ of $B m A C P$ dsRNA into newly emerged female moths. A dosedependent reduction in bombykol production was observed with 1,5 , and $10 \mu \mathrm{g}$ dsRNA injections resulting in bombykol levels 73,63 , and $59 \%$ of the control level, respectively (Figure 5B). To confirm the knockdown effect by $B m A C P$, we also examined other dsRNAs corresponding to non-overlapping $5^{\prime}$ and $3^{\prime}$ regions

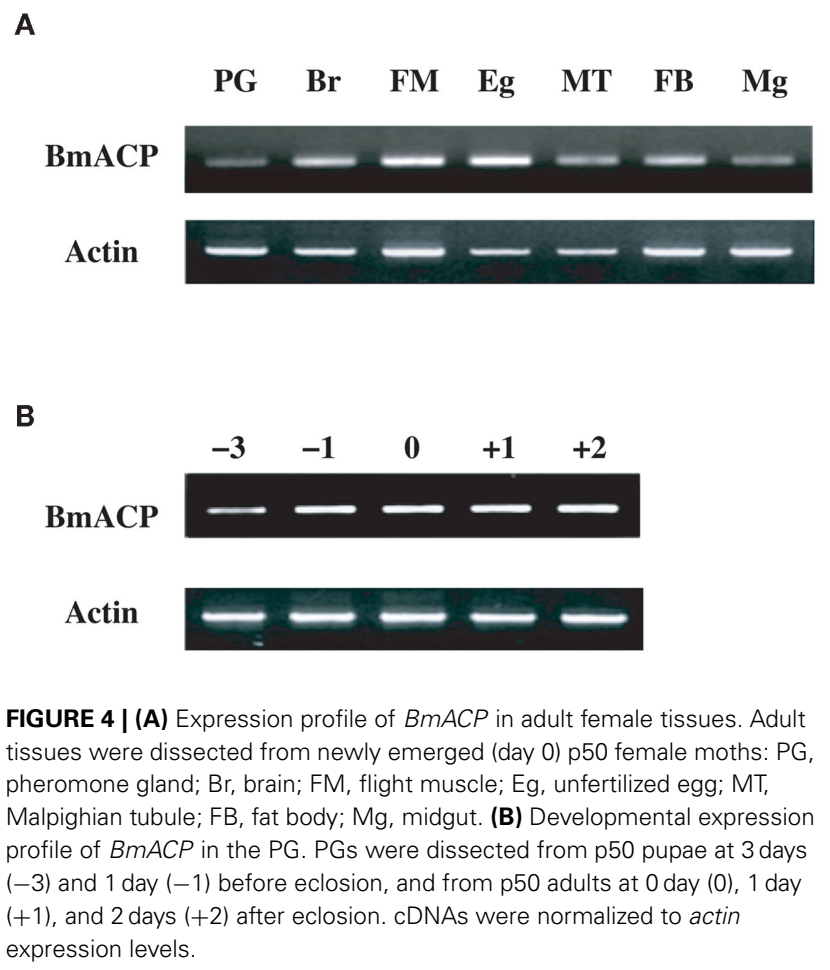

of BmACP; these dsRNAs had a similar knockdown effect on bombykol production (data not shown).

\section{IN VIVO EFFECT OF RNAi-MEDIATED BmACP KNOCKDOWN ON CYTOPLASMIC LD DYNAMICS}

We further examined the knockdown effect of BmACP on LD dynamics during pheromonogenesis (Figure 6). The most obvious characteristic feature of PG cells is the abundance of LDs within the cytoplasm (Fónagy et al., 2000, 2001). The role of the LDs in bombykol biosynthesis is to store the bombykol precursor fatty acid in the form of TAGs and release it in response to the external signal of PBAN (Matsumoto et al., 2002). To monitor the LD dynamics during pheromonogenesis, we have previously stained the LDs with the fluorescent lipid marker, Nile Red, and found that they appear 1-2 days before eclosion, rapidly 


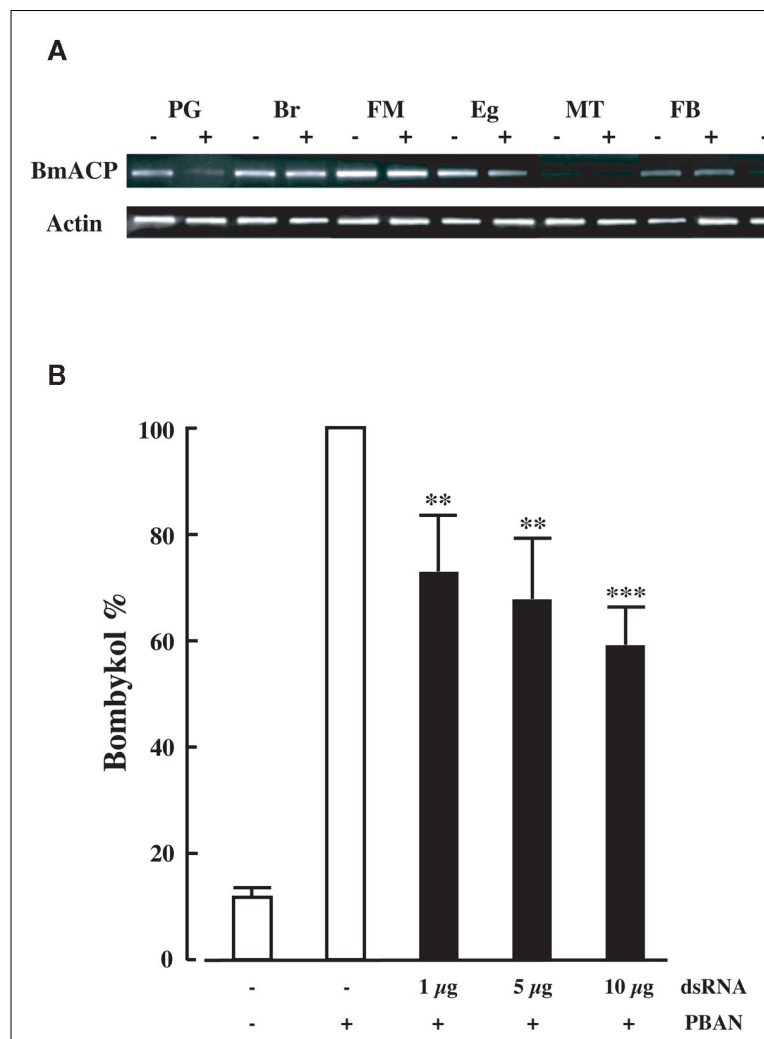

FIGURE 5 | Effect of RNAi treatment on in vivo bombykol production. (A) RNAi-induced suppression of BmACP transcripts. RT-PCR was performed using cDNAs generated from total RNA prepared from pupae injected with $0 \mu \mathrm{g}(-)$ or $5 \mu \mathrm{g}(+)$ dsRNAs for BmACP. Tissues were dissected from newly emerged (day 0) p50 female moths: PG, pheromone gland; $\mathrm{Br}$, brain; FM, flight muscle; Eg, unfertilized egg; MT, Malpighian tubule; FB, fat body; Mg, midgut. (B) Effect of RNAi treatment on in vivo bombykol production. Newly emerged female moths were injected with 1 , 5 , and $10 \mu \mathrm{g}$ dsRNA for BmACP. Results are expressed relative to the mean value for bombykol levels in non-RNAi-treated females following injection with 5 pmol PBAN $\left({ }^{* *} p<0.01 ;{ }^{* * *} p<0.001\right)$. Bars represent mean values $\pm \mathrm{SD}$ from independent experimental animals $(n=>12)$.

accumulate on the day of eclosion, and decrease in size and number after eclosion in response to PBAN (Fónagy et al., 2001). In RNAi-untreated controls, female moths accumulated numerous large LDs in the PG cell, which underwent a striking reduction following PBAN-induced LD lipolysis (Figure 6A,a,b, also see Ohnishi et al., 2009, 2011). In contrast, PGs of female moths treated with $B m A C P$ dsRNA accumulated noticeably smaller LDs (Figure 6A,a,c). Because these LDs store the bombykol precursor in the form of TAGs (14), we further examined the effects of BmACP knockdown on the fluctuation of total TAGs. Following extraction with acetone from dissected PGs, total TAG levels were measured by normal-phase HPLC on a PEGASIL-Silica column (Matsumoto et al., 1990). In BmACP knocked-down PGs, PBANinduced TAG lipolysis was comparable to that of the non-RNAitreated control (Figure 6B,b,d). In contrast, accumulation of TAG content was significantly reduced (38\%) in BmACP-knockdown PGs compared to the RNAi-untreated control (Figure 6B,a,c). Taken together, these results indicate that BmACP is integral to

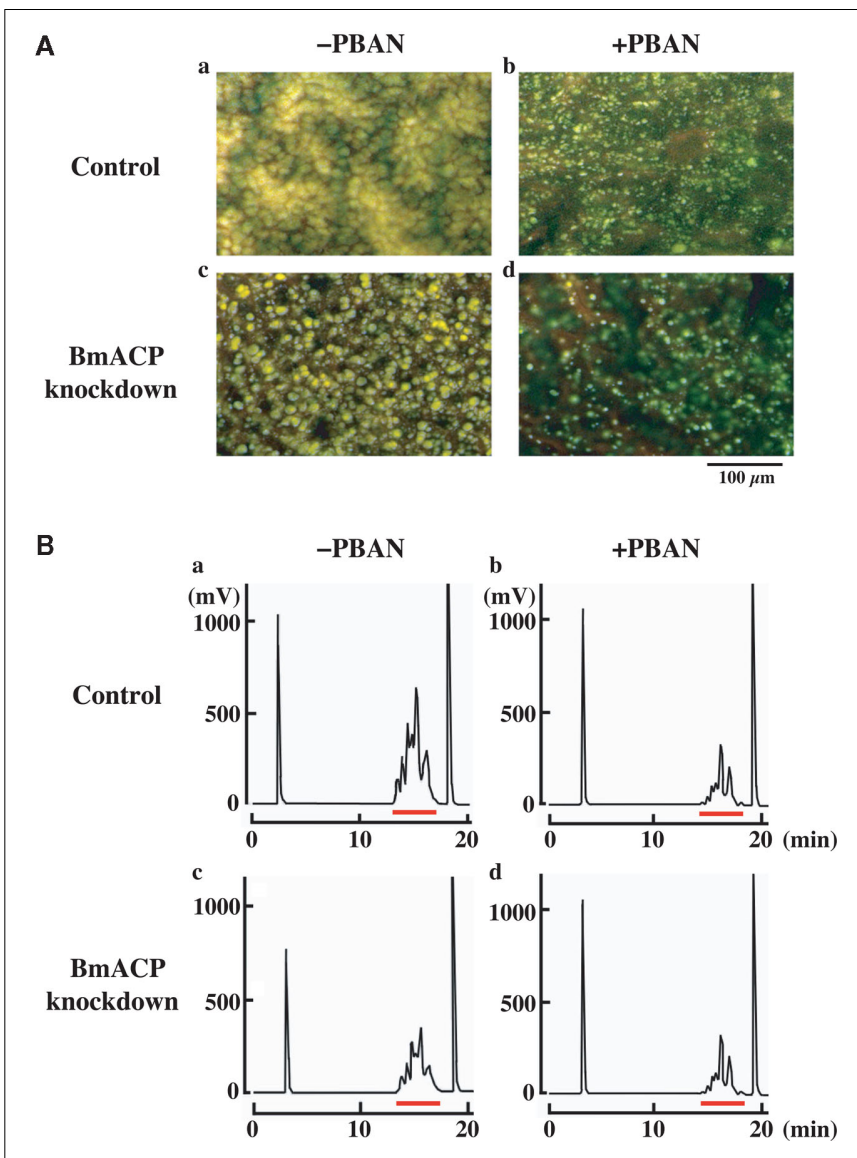

FIGURE 6 | Effect of RNAi-mediated knockdown of BmACP on in vivo LD dynamics. (A) Effect of RNAi treatment on cytoplasmic LD dynamics. PGs were dissected from 1-day-old decapitated female moths previously injected with either DEPC-treated $\mathrm{H}_{2} \mathrm{O}$ (control) or $10 \mu \mathrm{g} \mathrm{BmACP}$ dsRNA (BmACP knockdown) shortly after pupation. After eclosion, females were decapitated within $3 \mathrm{~h}$ of emergence, maintained at $25^{\circ} \mathrm{C}$ for $24 \mathrm{~h}$, and then injected with PBS alone (-PBAN) or 5 pmol B. mori PBAN (+PBAN). PBAN was injected three times at 3-h intervals. Cytoplasmic LDs were stained with the fluorescent lipid marker, Nile Red. Magnifications, 400×. (B) Effect of RNAi treatment on total TAG contents of the cytoplasmic LDs. One-day-old pupae were injected with DEPC-treated $\mathrm{H}_{2} \mathrm{O}$ (control) or $10 \mu \mathrm{g}$ BmACP dsRNA (BmACP knockdown). After eclosion, females were decapitated within $3 \mathrm{~h}$ of emergence, maintained at $25^{\circ} \mathrm{C}$ for $24 \mathrm{~h}$, and then injected with PBS alone (-PBAN) or 5 pmol of B. mori PBAN (+PBAN). PBAN was injected three times at 3-h intervals. Five PGs were extracted with acetone $90 \mathrm{~min}$ after PBAN injection, and the extract was subjected to HPLC on a PEGASIL-Silica column (Matsumoto et al., 2002). The relevant TAG fractions are underlined in red; TAGs were detected using an evaporative light scattering detector (ELSD).

the bombykol biosynthetic pathway in that it plays an essential role in accumulating the bombykol precursor for TAG storage in the cytoplasmic LDs before eclosion.

\section{IN VIVO EFFECT OF RNAi-MEDIATED BMACP KNOCKDOWN ON DE NOVO SYNTHESIS OF THE BOMBYKOL PRECURSOR}

The TAGs that compose cytoplasmic LDs in the PGs comprise various TAG species in which the bombykol precursor is predominantly sequestered at the $s n-1$ and/or $s n-3$ position as a major 
fatty acyl component (Matsumoto et al., 2002). Consequently, we sought to assess the effects of BmACP silencing on de novo synthesis of the bombykol precursor via the conventional FAS pathway. In agreement with our previous results (Matsumoto et al., 2002) and Figure 7, overall TAG contents in PGs of both control and RNAitreated moths were dramatically reduced in response to PBAN (Figures 6B and 7). In addition, the total amount of TAGs in RNAi-treated PGs was again reduced $\sim 34 \%$ compared to that of the non-RNAi-treated PGs (Figures 7A,C). Furthermore, there was a significant difference in the TAG elution profiles between non-RNAi-treated and RNAi-treated PGs before PBAN injection; silencing BmACP brought about a striking reduction in specific TAG peaks (see peaks 1, 1a, 2, 3 in Figure 7). MS analysis indicated that the TAG species eluted in these peaks are mainly comprised of the bombykol precursor fatty acid (Table 2; Matsumoto et al., 2002).

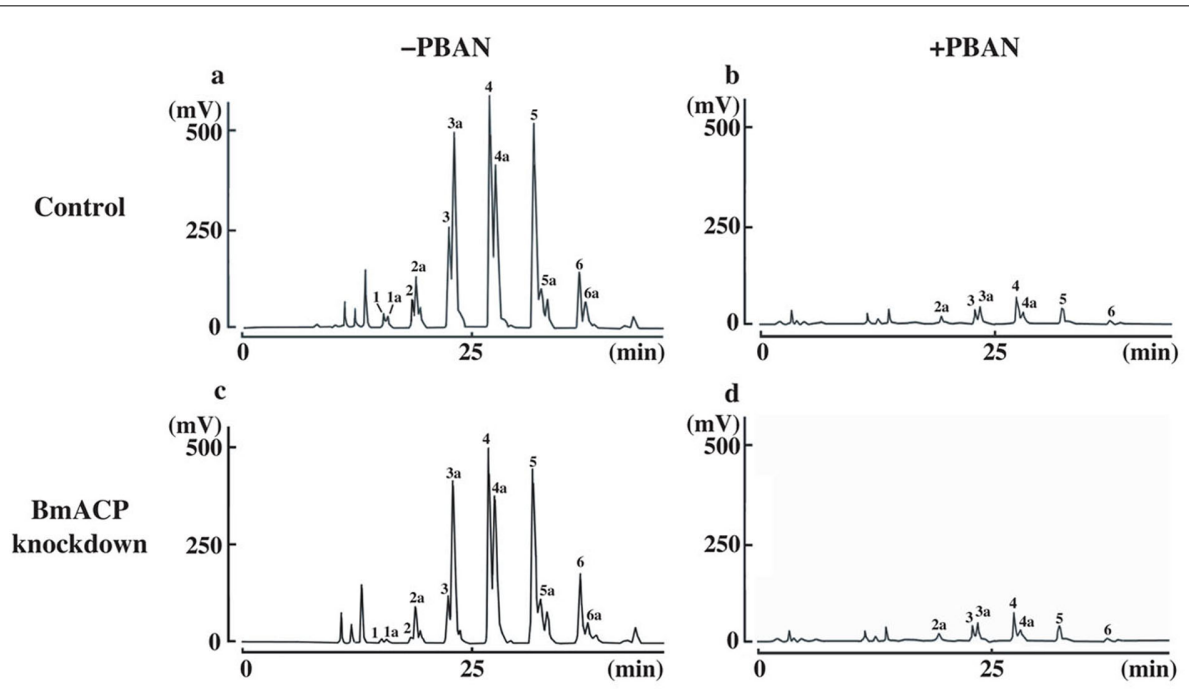

FIGURE 7 | Separation of PG-derivedTAG components. PG cells were dissected from 1-day-old decapitated female moths previously injected with either DEPC-treated $\mathrm{H}_{2} \mathrm{O}$ (control) or $10 \mu \mathrm{g}$ BmACP dsRNA (BmACP knockdown) shortly after pupation. After eclosion, females were decapitated within $3 \mathrm{~h}$ of emergence, maintained at $25^{\circ} \mathrm{C}$ for $24 \mathrm{~h}$, and then injected with PBS alone (-PBAN) or 5 pmol B. mori PBAN (+PBAN).
PBAN was injected three times at 3-h intervals. Five PGs were extracted with acetone 90 min after PBAN injection and the extracts were subjected to HPLC on a $\mathrm{C}_{22}$ docosil column (Matsumoto et al., 2002). Peaks were detected by ELSD and the fatty acyl composition of the TAG peaks was identified by FAB-MS and MS-MS analyses as described previously (Matsumoto et al., 2002).

Table 2 | Comparison of peak areas in Figures 7A,C.

\begin{tabular}{|c|c|c|c|c|c|}
\hline Peak $^{\#}$ & $\begin{array}{l}\text { Control (a) } \\
\text { Avg. peak area (AU) SD }\end{array}$ & $\begin{array}{l}\text { BmACP knockdown }(c) \\
\text { Avg. peak area (AU) SD }\end{array}$ & $\begin{array}{l}\text { Ratio (\%) } \\
B / A \times 100\end{array}$ & Student's $t$-test ${ }^{a}$ & Fatty acyl compositions ${ }^{b}$ \\
\hline 1 & $143 \pm 8$ & $50 \pm 6$ & 35.0 & *** & (C16:2, C16:2, C16:2) \\
\hline $1 a$ & $134 \pm 3$ & $57 \pm 11$ & 42.2 & $* * *$ & (C16:2, C16:2, C18:3) \\
\hline 2 & $211 \pm 12$ & $118 \pm 21$ & 55.6 & $* * *$ & (C16:2, C18:2, C16:2) \\
\hline $2 a$ & $941 \pm 166$ & $678 \pm 155$ & 72.0 & * & (C16:1, C18:3, C18:3) and (C16:2, C18:2, C18:3) \\
\hline 3 & $1,622 \pm 204$ & $492 \pm 62$ & 30.3 & $* * *$ & (C16:2, C18:1, C16:2) \\
\hline $3 a$ & $2,022 \pm 318$ & $1,691 \pm 270$ & 80.1 & * & (C16:2, C18:1, C18:3) \\
\hline 4 & $2,169 \pm 296$ & $1,550 \pm 329$ & 71.5 & ** & (C16:2, C18:1, C16:1) and/or (C16:1, C18:1, C16:2) \\
\hline $4 a$ & $1,831 \pm 318$ & $1,333 \pm 270$ & 72.8 & * & $\begin{array}{l}\text { (C16:1, C18:1, C18:3), (C16:1, C18:2, C18:2), and } \\
\text { (C16:2, C18:1, C18:2) }\end{array}$ \\
\hline 5 & $2,140 \pm 248$ & $1,638 \pm 399$ & 76.5 & * & (C16:2, C18:1, C18:1) and/or (C18:1, C18:1, C16:2) \\
\hline $5 a$ & $689 \pm 67$ & $595 \pm 116$ & 86.4 & NS & (C18:1, C18:1, C18:3) and (C18:1, C18:2, C18:2) \\
\hline 6 & $624 \pm 72$ & $552 \pm 42$ & 88.5 & NS & (C16:1, C18:1, C18:1) and/or (C18:1, C18:1, C16:1) \\
\hline $6 a$ & $301 \pm 49$ & $221 \pm 45$ & 73.3 & NS & (C18:1, C18:1, C18:2) \\
\hline
\end{tabular}

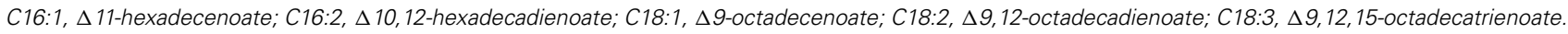
NS, not significant.

a Statistical analysis of avg. peak area control samples vs. BmACP knockdown samples performed using Student's t-test ( $n=5$ ).

${ }^{b}$ Fatty acyl compositions of the TG(s) in each peak were identified with Fast Atom Bombardment MS and MS-MS analyses (Matsumoto et al., 2002).

${ }^{*} p<0.05,{ }^{* *} p<0.01,{ }^{* *} p<0.001$. 


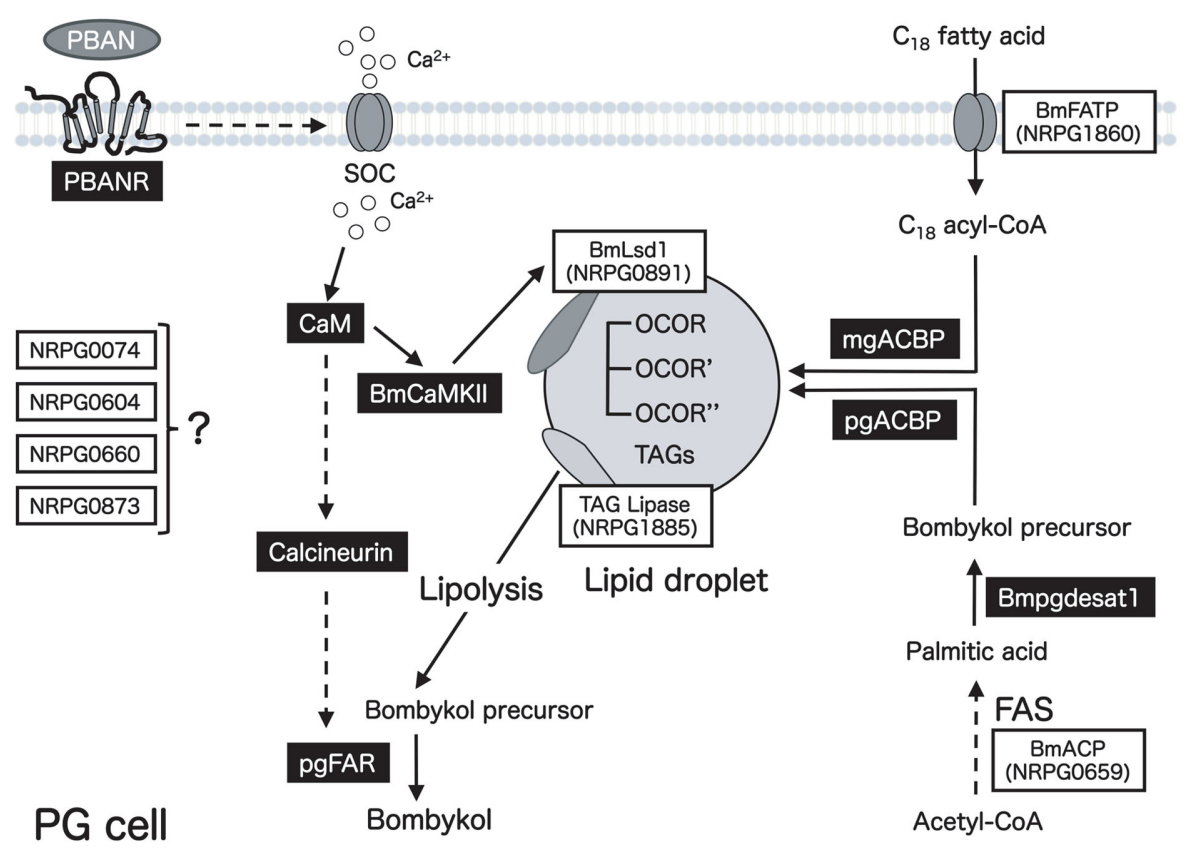

FIGURE 8 | Schematic diagram of the proposed bombykol biosynthetic pathway. Eight EST clones involved in bombykol biosynthesis are indicated in boxes. Gene products that have been characterized are indicated in black boxes. Potential sites of regulation are indicated by dashed lines.

\section{DISCUSSION}

We have developed a method to facilitate identification of the genes involved in bombykol biosynthesis, using RT-PCR expression analysis and RNAi-mediated in vivo gene silencing in conjunction with EST databases. In the current study, we identified eight EST clones that likely encode various proteins that play a role in bombykol production (Table 1; Figure 1). These eight EST clones include two clones that encode BmFATP and BmLsd1 (NRPG1860 and NRPG0891), two proteins that we have recently identified and shown to be functionally associated with LD dynamics during B. mori pheromonogenesis (Ohnishi et al., 2009, 2011). We have reported that BmFATP plays a role in stimulating the TAG synthesis required for LD accumulation via a process similar to the so-called vectorial acylation that couples the uptake of extracellular fatty acids with activation to CoA thioesters (Ohnishi et al., 2009). In contrast, BmLsd1 plays an essential role in the TAG lipolysis associated with bombykol biosynthesis via phosphorylation with activation of BmCaMKII by influx of extracellular $\mathrm{Ca}^{2+}$ (Ohnishi et al., 2011).

Of the eight clones identified in the current study, we further characterized the clone that encodes BmACP. ACP is a highly conserved 70-100-aa carrier of acyl intermediates during FAS (Byers and Gong, 2007). In yeast and mammals, ACP exists as a separate domain within a large multifunctional fatty acid synthase polyprotein (type I FAS), whereas it is a small monomeric protein in bacteria and plastids (type II FAS). Because many insect sex pheromones are synthesized de novo from acetyl-CoA via the conventional long chain FAS pathway within PG cells (Bjostad et al., 1987; Jurenka, 2003), identification of BmACP in the PG suggests that it plays a significant role in the early steps of bombykol biosynthesis. Accordingly, RNAi-mediated gene silencing of BmACP in vivo resulted in a significant reduction in bombykol production, suggesting the functional relevance of BmACP in bombykol biosynthesis (Figure 5). In addition, RNAi treatment resulted in accumulation of noticeably smaller LDs in the PG (Figure 6), indicating that it plays an essential role in $\mathrm{LD}$ accumulation prior to eclosion. While similar results was observed when BmFATP gene expression was suppressed (Ohnishi et al., 2009), the analysis of TAG contents revealed a significant difference. BmACP suppression mainly reduced the TAGs containing the bombykol precursor fatty acid, whereas the BmFATP suppression mainly reduced the TAGs containing the essential $\mathrm{C}_{18}$-fatty acids derived from diet (Figure 7 ). These results are consistent with the general properties of ACPs and strongly suggest that BmACP plays an essential role in the biosynthesis of the bombykol precursor palmitic acid via the canonical FAS pathway in PG cells during pheromonogenesis. These results also indicate that BmACP and BmFATP function cooperatively to populate the TAGs comprising the cytoplasmic LDs (Figure 8). In addition, our present results validate the methodological approach we have taken to mine EST databases for unidentified functional molecules that play a critical role in moth sex pheromone production.

\section{ACKNOWLEDGMENTS}

We thank Shinji Atsusawa, Masaaki Kurihara, and Katsunori Ichinose for maintaining the B. mori colony. This work was supported by the Lipid Dynamics Research Program from RIKEN and the Targeted Proteins Research Program (TPRP) from the Ministry of Education, Culture, Sports, Science, and Technology of Japan. 


\section{REFERENCES}

Bjostad, L. B., Wolf, W. A., and Roelofs, W. L. (1987). "Pheromone biosynthesis in lepidopterans: desaturation and chain shortening," in Pheromone Biochemistry, eds G. D. Prestwich and G. J. Blomquist (Orlando, FL: Academic Press), 77-120.

Byers, D. M., and Gong, H. (2007). Acyl carrier protein: structure-function relationships in a conserved multifunctional protein family. Biochem. Cell Biol. 85, 649-662.

Chomczynski, P., and Sacchi, N. (1987). Single-step method of RNA isolation by acid guanidinium thiocyanatephenol-chloroform extraction. Anal. Biochem. 162, 156-159.

Fónagy, A., Matsumoto, S., Uchiumi, K., Orikasa, C., and Mitsui, T. (1992). Action of pheromone biosynthesis activating neuropeptide on pheromone glands of Bombyx mori and Spodoptera litura. J. Pest Sci. 17, 47-54.

Fónagy, A., Yokoyama, N., and Matsumoto, S. (2001). Physiological status and change of cytoplasmic lipid droplets in the pheromoneproducing cells of the silkmoth, Bombyx mori (Lepidoptera, Bombycidae). Arthropod Struct. Dev. 30, 113-123.

Fónagy, A., Yokoyama, N., Okano, K., Tatsuki, S., Maeda, S., and Matsumoto, S. (2000). Pheromoneproducing cells in the silkmoth, Bombyx mori: identification and their morphological changes in response to pheromonotropic stimuli. J. Insect Physiol. 46, 735-744.

Hull, J. J., Lee, J. M., Kajigaya, R., and Matsumoto, S. (2009). Bombyx mori homologs of STIM1 and Orail are essential components of the signal transduction cascade that regulates sex pheromone production. J. Biol. Chem. 284, 31200-31213.

Hull, J. J., Lee, J. M., and Matsumoto, S. (2010). Gq $\alpha$-linked phospholipase $C \beta 1$ and phospholipase $C \gamma$ are essential components of the pheromone biosynthesis activating neuropeptide (PBAN) signal transduction cascade. Insect Mol. Biol. 19, 553-566.

Hull, J. J., Ohnishi, A., Moto, K., Kawasaki, Y., Kurata, R., Suzuki,
M. G., and Matsumoto, S. (2004). Cloning and characterization of the pheromone biosynthesis activating neuropeptide receptor from the Silkmoth, Bombyx mori: Significance of the carboxyl terminus in receptor internalization. J. Biol. Chem. 279, 51500-51507.

Jurenka, R. A. (2003). "Biochemistry of female moth sex pheromones," in Insect Pheromone Biochemistry and Molecular Biology, eds G. J. Blomquist and R. G. Vogt (Oxford: Elsevier Academic Press), 53-80.

Kitamura, A., Nagasawa, H., Kataoka, H., Inoue, T., Matsumoto, S., Ando, T., and Suzuki, A. (1989). Amino acid sequence of pheromonebiosynthesis-activating neuropeptide (PBAN) of the silkworm, Bombyx mori. Biochem. Biophys. Res. Commun. 163, 520-526.

Matsumoto, S., Fónagy, A., Yamamoto, M., Wang, F., Yokoyama, N., Esumi, Y., and Suzuki, Y. (2002). Chemical characterization of cytoplasmic lipid droplets in the pheromoneproducing cells of the silkmoth, Bombyx mori. Insect Biochem. Mol. Biol. 32, 1447-1455.

Matsumoto, S., Hull, J. J., Ohnishi, A., Moto, K., and Fónagy, A. (2007). Molecular mechanisms underlying sex pheromone production in the silkmoth, Bombyx mori: characterization of the molecular components involved in bombykol biosynthesis. J. Insect Physiol. 53, 752-759.

Matsumoto, S., Kitamura, A., Nagasawa, H., Kataoka, H., Orikasa, C., Mitsui, T., and Suzuki, A. (1990). Functional diversity of a neurohormone produced by the suboesophageal ganglion: molecular identity of melanization and reddish coloration hormone and pheromone biosynthesis activating neuropeptide. J. Insect Physiol. 36, 427-432.

Matsumoto, S., Ohnishi, A., Lee, J. M., and Hull, J. J. (2010). Unraveling the pheromone biosynthesis activating neuropeptide (PBAN) signal transduction cascade that regulates sex pheromone production in moths. Vitam. Horm. 83, 425-445.

Matsumoto, S., Yoshiga, T., Yokoyama, N., Iwanaga, M., Koshiba, S., Kigawa,
T., Hirota, H., Yokoyama, S., Okano, K., Mita, K., Shimada, T., and Tatsuki, S. (2001). Characterization of acyl-CoA-binding protein (ACBP) in the pheromone gland of the silkworm, Bombyx mori. Insect Biochem. Mol. Biol. 31, 603-609.

Moto, K., Suzuki, M. G., Hull, J. J., Kurata, R., Takahashi, S., Yamamoto, M., Okano, K., Imai, K., Ando, T., and Matsumoto, S. (2004). Involvement of a bifunctional fatty-acyl desaturase in the biosynthesis of the silkmoth, Bombyx mori, sex pheromone. Proc. Natl. Acad. Sci. U.S.A. 101, 8631-8636.

Moto, K., Yoshiga, T., Yamamoto, M., Takahashi, S., Okano, K., Ando, T., Nakata, T., and Matsumoto, S. (2003). Pheromone gland-specific fatty-acyl reductase of the silkmoth, Bombyx mori. Proc. Natl. Acad. Sci. U.S.A. 100, 9156-9161.

Ohnishi, A., Hashimoto, K., Imai, K., and Matsumoto, S. (2009). Functional characterization of the Bombyx mori fatty acid transport protein (BmFATP) within the silkmoth pheromone gland. J. Biol. Chem. 284, 5128-5136.

Ohnishi, A., Hull, J. J., Kaji, M. Hashimoto, K., Lee, J. M., Tsuneizumi, K., Suzuki, K., Dohmae, N., and Matsumoto, S. (2011). Hormone signaling linked to silkmoth sex pheromone biosynthesis involves $\mathrm{Ca}^{+} /$calmodulin-dependent protein kinase II (CaMKII)-mediated phosphorylation of the insect PAT family protein Bombyx mori lipid storage droplet protein-1 (BmLsd1). J. Biol. Chem. 286, 24101-24112.

Ohnishi, A., Hull, J. J., and Matsumoto, S. (2006). Targeted disruption of genes in the Bombyx mori sex pheromone biosynthetic pathway. Proc. Natl. Acad. Sci. U.S.A. 103, 4398-4403.

Ozawa, R., and Matsumoto, S. (1996). Intracellular signal transduction of PBAN action in the silkworm, Bombyx mori: involvement of acyl CoA reductase. Insect Biochem. Mol. Biol. 26, 259-265.

Percy, J. E., and Weatherston, J. (1974). "Gland structure and pheromone production in insects," in Pheromones, ed. M. C. Birch
(Amsterdam: North-Holland Publishing Co.), 11-34.

Rafaeli, A. (2002). Neuroendocrine control of pheromone biosynthesis in moths. Int. Rev. Cytol. 213, 49-91.

Rafaeli, A. (2009). Pheromone biosynthesis activating neuropeptide (PBAN): regulatory role and mode of action. Gen. Comp. Endocrinol. 162, 69-78.

Rafaeli, A., and Jurenka, R. A. (2003). "PBAN regulation of pheromone biosynthesis in female moths," in Insect Pheromone Biochemistry and Molecular Biology, eds G. J. Blomquist and R. G. Vogt (Oxford: Elsevier Academic Press), 107-136.

Raina, A. K., Jaffe, H., Kempe, T. G., Keim, P., Blacher, R. W., Riley, C. T., Klun, J. A., Ridgway, R. L., and Hayes, D. K. (1989). Identification of a neuropeptide hormone that regulates sex pheromone production in female moths. Science 244, 769-798.

Yoshiga, T., Okano, K., Mita, K., Shimada, T., and Matsumoto, S. (2000). cDNA cloning of acyl-CoA desaturase homologs in the silkworm, Bombyx mori. Gene 246, 339-345.

Conflict of Interest Statement: The authors declare that the research was conducted in the absence of any commercial or financial relationships that could be construed as a potential conflict of interest.

Received: 26 September 2011; accepted: 18 November 2011; published online: 07 December 2011.

Citation: Ohnishi A, Kaji M, Hashimoto K and Matsumoto S (2011) Screening for the genes involved in bombykol biosynthesis: identification and functional characterization of Bombyx mori acyl carrier protein. Front. Endocrin. 2:92. doi: 10.3389/fendo.2011.00092

This article was submitted to Frontiers in Experimental Endocrinology, a specialty of Frontiers in Endocrinology.

Copyright (C) 2011 Ohnishi, Kaji, Hashimoto and Matsumoto. This is an open-access article distributed under the terms of the Creative Commons Attribution Non Commercial License, which permits non-commercial use, distribution, and reproduction in other forums, provided the original authors and source are credited. 